\title{
Customer satisfaction with clinical laboratory services provided by the Ethiopian health facilities: a systematic review and meta-analysis
}

Teshiwal Deress ( $\nabla$ teshiwalderess@gmail.com )

University of Gondar, College of Medicine and Health Sciences, School of Biomedical and Laboratory

Sciences https://orcid.org/0000-0002-1678-604X

Yihenew Million

University of Gondar

Teshome Belachew

University of Gondar

Mekonnen Girma

University of Gondar

\section{Research}

Keywords: Clinical laboratory, Quality service, Customer satisfaction, Meta-analysis, Ethiopia

Posted Date: September 1st, 2020

DOI: https://doi.org/10.21203/rs.3.rs-65567/v1

License: (c) (i) This work is licensed under a Creative Commons Attribution 4.0 International License. Read Full License 


\section{Abstract \\ Background}

Medical laboratory helps health professionals and patients with disease diagnosis and treatment monitoring. While providing services, maintaining customer satisfaction is an essential determinant of the success and long-term survival of the facility. So far, several studies conducted in Ethiopia on the evaluation of laboratory customer satisfaction; however, the level of satisfaction was significantly differed from study to study. Therefore, this study aimed to provide an estimated pooled satisfaction level of clinical laboratory customers with laboratory services in Ethiopia.

\section{Methods}

Online electronic databases, including Science Direct, Medline, HINARI, TRIP database, and African Journals Online, were searched. The pooled estimate of laboratory service satisfaction was determined using the random-effects model due to the presence of considerable heterogeneity among studies. The possible sources of heterogeneity were analyzed using subgroup analysis, sensitivity analysis, and metaregression.

\section{Results}

The analysis of 18 Full text articles showed that the level of clinical laboratory service satisfaction among Ethiopian laboratories ranged from $48-91 \%$. The pooled estimate was $66 \%(95 \% \mathrm{Cl}: 59-73)$. Among the moderator variables, sample size $(p=0.34)$ and year of publication $(p \leq 0.01)$ of the included studies showed statistically significant associations with the level of service satisfaction. The sensitivity analysis declares no influence on the overall effect estimate while removing a single study from the analysis at a time. The statistical tests declare the absence of publication bias.

\section{Conclusions}

In Ethiopia, the pooled level clinical laboratory service satisfaction was low. The year of publication and sample size showed a statistically significant relationship with service satisfaction. Strengthening the scope of existing diagnostic services, implementing immediate corrective actions for the unsatisfied customers, and having a mechanism of continuous monitoring of laboratory activities should be implemented to assure adequate service quality. Generally, the study can provide a current estimate that could be valuable for policymakers.

\section{Background}


The clinical laboratory is an integral component of the healthcare system [1]. It helps health professionals with disease diagnosis, guiding treatment, determining drug resistance, disease prevention, and disease control, identifying diseases of public health significance, and contributed to the public health policy development [1-3]. It has different customers (patients, health professionals, public health authorities, and others who would like to get and use laboratory data for the community health) whose opinions are vital components in providing laboratory managers with opportunities to identify areas of improvement [4]. Satisfaction is the degree to which the customers feel their needs fulfilled by the service provider $[5,6]$. Customer satisfaction as an essential determinant of success and long-term survival of the healthcare industry has caught the providers' attention in the present competitive conditions [7-9]. Evaluating to what extent clinical laboratory customers are satisfied with laboratory services is a vital quality domain, as satisfied patients are more likely to comply and continue using medical care services and stay within a health provider [10].

On the other hand, clients who are not satisfied with the laboratory services are more likely to discontinue the health services and may have worse outcomes because they miss appointments and live against the advice or fail to follow treatment plans [11]. In most cases, the mismatch between patient expectations and the services they received leads dissatisfaction. Clinical laboratory service satisfaction is influenced by the level of quality service delivered, staff professionalism, provision of the adequate information system, availability of ordered tests, length of waiting time to obtain laboratory results, location of laboratory room, and availability and/or accessibility of good latrine [12]. The problems are aggravated mainly due to the lack of adequately designed laboratory rooms, shortage of short term and long term training for laboratory staff, lack of water and electricity, shortage of equipment and supplies, the absence of adequate maintenance and spare parts and lack of follow-up and supervision [13]. Monitoring laboratory customers' satisfaction is an essential and useful tool required for laboratory quality improvement as well as to get accreditation [14]. Customer satisfaction with medical laboratory services is the most important quality indicators in laboratory medicine that reflects the service provider's ability to successfully meet the customers' expectations and needs [15]. It also facilitates the identification of problem areas and generates ideas for resolving these problems [16]. Customer satisfaction is a significant component of a quality management system (QMS) and a considerable focus of the International Standardization for Organization (ISO) standards [5], Joint Commission on Accreditation of Healthcare Organizations (JCAHO), and the College of American Pathologists (CAPs) Laboratory Accreditation Programs. These organizations require clinical laboratories to measure the level of their customer satisfaction as part of their respective accreditation programs at least once every two years inspection cycle $[7,17]$. Measurement of customer satisfaction brings necessary customer preferences into the quality assessment process through which mistaken assumptions can be corrected and enable to focus on customers value most elements [18].

\section{Methods}

\section{Study design and protocol registration}


The protocol of the current systematic review and meta-analysis was designed following the "Preferred Reporting Items for Systematic Reviews and Meta-Analysis Protocols" (PRISMA-P 2015) guidelines [19] and prospectively registered in the PROSPERO database with the protocol registration number of CRD42020187022.

\section{Study setting}

This review was conducted in Ethiopia, which is a highly populated country in eastern Africa. Currently, the population is estimated to be more than a hundred million. Because of the rapid growth of the population, the number of healthcare facilities is significantly increasing $[20,21]$. Currently, the healthcare facilities are grouped into three major categories, including primary, secondary, and tertiary levels. Earlier in 2011 , a total of 22,792 health facilities were registered in the country to provide different health services for the population in their catchment area. From this, about 125, 2999, 15,668, and 4000 contributed by hospitals, health centers, health posts, and private clinics, respectively [22]. The health centers and health posts provide primary healthcare services, and approximately 40000 and 3000-5000 population, respectively, is allocated for them. Likewise, primary hospitals were established to serve about 60000-10000 population. General and specialized hospitals cover a wide area, and they mainly provide specialized and referral services for 1 to 5 million population [23]. Currently, with the rapid increment of health facilities, the ratio of the healthcare worker to the health facilities is still inadequate [24].

\section{Article searching strategy}

Before starting the actual work, the PROSPERO database was searched to check the presence of similar projects related to this topic. Literature searching strategy, selection of eligible articles, data extraction, data analysis, and result reporting has done according to the PRISMA guidelines [25]. Articles searched from Science Direct, HINARI, Medline through PubMed, African Journals Online (AJOL), and TRIP database databases using a combination of keywords and Boolean functions the PubMed search string is attached as a supplementary file (Supplementary materials 1 ). All the mentioned databases searched in English without publication year restriction. The database-specific search strings were developed according to the database requirements. Besides, to include as many articles as possible, manual hand searching on google and Google Scholar was done. Moreover, reference lists of both included and excluded studies were screened. The most recent database search was done on April 1, 2020.

\section{Article selection, eligibility, and data extraction}

The searched studies imported into EndNote X9 software and duplicate articles were removed. Both authors screened the articles independently by title, abstract, and full-text to identify eligible studies. Studies were considered as eligible if they were primary studies and accessed in full-text format, conducted in Ethiopian settings, and published in English from peer-reviewed journals. Besides, studies with prevalence data clearly stated or if missed the presence of adequate data to calculate the prevalence (known sample size and number of satisfied customers) considered. The data abstraction form prepared in the Microsoft Excel Spreadsheet which includes; first author's name, year of study, publication year, region, type of health facility, study group, study design, sample size, sampling technique, and the number 
of study participants satisfied with the laboratory services. Both authors extracted the data independently, and any disagreement (inconsistency) was resolved by discussion.

\section{Quality assessment}

The quality assessment was done independently by authors using the Joanna Briggs Institute (JIB) quality assessment tool for prevalence studies [26]. The tool has nine quality domains with yes, no, unclear, and not applicable response options including; 1) appropriate sampling frame, 2) proper sampling technique, 3) sufficient sample size, 4) description of the study subject and setting, 5) appropriate data analysis, 6) use of valid methods, 7) use of valid measurement, 8) appropriate statistical analysis, and 9) adequate response rate [26]. Operationally, 1 and 0 values provided for yes and (no and unclear) responses, respectively. Finally, the composite and mean scores are computed. Studies with quality scores below the mean value and "mean score and above" were categorized as having a high and minimal risk of bias, respectively. The quality of data abstraction (inter-rater agreement) assessed using Cohen's Kappa, and the inter-reliability coefficient was found to be 0.784 ( $p<$ $0.001)$ that indicates excellent agreement.

\section{Data synthesis and analysis}

Data were analyzed using the malaprop program of STATA software, and the Freeman Tukey double arcsine transformation ( $\mathrm{ft}$ ) was enabled to include proportions close to 0 and 1 [27]; otherwise, those studies with 1 and 0 proportions could be omitted and lead to a biased estimate. This program computes the weighted pooled estimate and then perform back-transformation on the pooled estimate. The time transformed prevalence is weighted very slightly towards $50 \%$, which enables 0 prevalence studies included in the analysis [28]. When there is evidence of across study heterogeneity, the random-effects model is recommended for analysis [29]. In this case, the Dersimonian and Laird method is most used [30]. The presence of heterogeneity among studies checked using $I^{2}$ test statistics, which estimates the

presence of observed differences between-studies due to heterogeneity. The $I^{2}$ value can range from 0 to $100 \%$, and $0 \%$ indicates the absence of heterogeneity; whereas, $100 \%$ is a definitive indicator of significant heterogeneity. The $25 \%, 50 \%$, and $75 \%$ values represent low, medium, and high heterogeneity between studies, respectively [31]. In addition, a $p$-value of $<0.05$ is used to declare heterogeneity [32]. In this meta-analysis, the $\mathrm{I}^{2}$ value was high $(97.77 \%)$, which an indication of significant heterogeneity. Due to this reason, the analysis conducted using a random-effects model at $95 \% \mathrm{Cl}$ as opposed to the fixed effects model to adjust the observed variability among studies. The possible sources of heterogeneity are investigated through stratified analysis, sensitivity analysis, and meta-regression. Visual inspection of funnel plots and results of Egger's weighted statistics were used to investigate the presence of publication bias and small-study effects. All data management and statistical analysis performed using STATA software version 16.0 (Stator LLC College Station TX 77845, USA for windows version).

\section{Results}




\section{Study selection}

Initially, 455 studies were retrieved from databases and hand searching. From this, 98 studies were removed due to duplication. Then, 357 articles were screened by title/ abstract, and 333 studies were studies excluded because of being unrelated to the current review. The remaining 24 full-text articles were further refined, and 6 of them excluded due to studies being conducted in other countries, outpatient departments, inpatient departments, and emergency departments. Finally, 18 full-text articles fulfilled the inclusion criteria and included in the review [Figure 1].

\section{Characteristics of primary studies included in the review}

Eighteen full-text articles $[11,16,17,33-47]$ included in the systematic review and meta-analysis that included a total of 8495 study participants. The smallest and largest sample sizes of the studies were 105 and 2399, respectively [17, 42]. Similarly, the lowest and highest level of the laboratory customer satisfaction was $48.3 \%$ and $90.8 \%$, respectively $[45,46]$. All the studies conducted using a cross-sectional study design. The earliest and latest studies were conducted in 2011 and 2020, respectively [17, 40]. Information about clinical laboratory service satisfaction obtained from four regions and two selfadministrative cities. Among studies, about one third obtained from Addis Ababa city. No study was obtained from the Somali, Benishangul Gomez, Afar, and Gambelia regions. About two-thirds of the studies were conducted on primary service users (clients) [Table 1].

\section{Qualitative findings}

Patients were satisfied with the language laboratory professionals used for communication [33, 38, 45] the cost of laboratory services, the courtesy provided by the laboratory workers [34, 35, 41, 42], and availability of laboratory staff during working hours. However, they were dissatisfied with timeline of test results $[17,34,36,37,41,47]$, adequacy and quality of waiting area [33,37], the lack of adequate information on how to collect clinical specimens [35,40], bad blood collection processes (many needlestick attempts) [17], latrine cleanness [11, 16, 17, 33, 36, 37, 40, 45], lack of secure area to put personal utilities during sample collection [34,46], and timely notification of critical values [36, 41, 42] [Table 2].

\section{A meta-analysis of customer satisfaction rate with clinical laboratory services in Ethiopia}

After applying different selection criteria, eighteen studies were included in the qualitative synthesis and meta-analysis. Based on the random-effects model, the value of $\mathrm{I}^{2}$ is found to be $97.97 \%$ with $p \leq 0.01$, which is an indicator of considerable heterogony among studies. Due to this, the pooled estimate was conducted using the random-effects model. The level of laboratory service satisfaction ranged from $48 \%$ to $91 \%$, with a pooled estimate of $66 \%(95 \% \mathrm{Cl}$ : $59-73)$ [Figure 2]. So, when there is significant heterogeneity among the included studies should be analyzed using the random-effects model rather than the fixed effects model, and the possible sources of variability should deal using subgroup analysis or meta-regression as appropriate. 


\section{Investigation of heterogeneity}

The subgroup analysis was conducted on the year of publication, sample size, sampling technique, study participants, and study quality. The heterogeneity among the subgroups did not significantly change. The lowest level of $I^{2}(88.82 \%)$ was among studies conducted with a sample size of below 384 . The highest level of satisfaction (82\%) among the study participants was obtained from the studies conducted between 2011 and 2014. While the year of publication increases the level of customer satisfaction with clinical laboratory services is significantly decline [Table 3].

\section{Meta-regression and sensitivity analysis}

A meta-regression analysis was conducted to investigate the possible sources of heterogeneity. It is a preferred technique for investigating heterogeneity compared to subgroup analysis and has the advantage of running multiple covariates at the same time [48]. The sample size and year of publications of the included studies were considered as covariates. While increasing sample size, there is a slight increment of customer satisfaction with clinical laboratory services (coef $=0.02, p=0.34$ ). On the other hand, disapprovingly, when the publication year increases, the level of laboratory service satisfaction significantly decreases (coef $=-4.39, p \leq 0.01$ ) [Figure 3].

Further, a sensitivity analysis was performed by removing a single study from the analysis to ensure the stability of the overall effect estimate. The result indicated that removing a single study from the analysis did not significantly influence the pooled estimate. The pooled effect estimate of laboratory service satisfaction ranged from $63.3 \%, 95 \% \mathrm{Cl}$ : $31.87-94.78$ (if Belay $\mathrm{M}$. et al. is excluded) [45] to $77.0 \% 95 \% \mathrm{Cl}$ : 44.13-109.83 (if Alelign A. et al. is excluded from the analysis) [46]. The analysis of the sample sizes showed no influence was observed on the overall effect estimate while removing a single study at a time from the analysis [Figure 4].

\section{Publication bias}

The presence of publication bias was evaluated using the funnel plot and objectively by Egger's and Begg's statistical tests. Each point in the funnel plot stands for a single study, an asymmetrical distribution of the points is indicative of the presence of publication bias [49]. Publication bias occurs when published studies do not represent all the studies conducted. The funnel plot showed minimal evidence of publication bias. However, after conducting statistical tests, results showed no statistically significant publication bias (Egger's $p=0.21$ and Begg's test $p=0.08$ ) [Figure 5].

\section{Discussion}

Customer satisfaction with clinical laboratory services is a valuable tool to evaluate the degree to which how much the laboratory organization meets it's user preferences. Dissatisfaction with services can result in early withdrawal and significantly affect the long-term survival of the healthcare industry in general. It is also an essential component of the laboratory accrediting organization requirements. The level of 
laboratory service satisfaction rate among the included studies in Ethiopia is highly variable, ranging from $48-91 \%$, with an estimated pooled satisfaction level of $66 \%(95 \% \mathrm{Cl}: 59-73)$.

Customer satisfaction is one of the most indicators of successful laboratory service provision, and it serves as a vital quality improvement tool. Thus, identifying factors associated with the level of satisfaction can support health service managers to design and implement the proper intervention for improvement. With this regard, though there was an adequate level of satisfaction among some studies about security and confidentiality of laboratory test results, customers among most studies were less likely satisfied with the confidentiality of their information. In some cases, patients were highly satisfied with the courtesy given by the laboratory professionals; however, the lack of prompt delivery of test results, particularly notification of critical data identified for feature improvement. The laboratory customers were less likely satisfied due to the provision of inadequate information during the specimen collection process, unavailability of laboratory workers during the working hours, and the lack of secure area to put personal utilities. Besides, bad clinical practices such as many needlestick attempts during blood collection processes, missing test results, cost of the laboratory services, and the lack of provision of adequate information on how to follow when using the laboratory services found as a source of complaint by the customers. Also, the location of the laboratory building within the health facility and the lack of adequate sitting arrangements around the waiting areas were less likely suitable for the laboratory customers. Further, the accessibility, hygienic status, and comfort of latrines has been identified as a significant problem for most of the laboratory services in Ethiopia, which needs adequate attention given by the health facility administrators, particularly by the laboratory heads and section supervisors.

The subgroup analysis showed no significant decline in the level of heterogeneity among the included studies in the meta-analysis. However, the lowest and highest level of satisfaction was noted among lowquality studies and studies conducted from 2011-2013. Regarding the associated factors, when the sample size increases, the level of laboratory service satisfaction slightly increases though the $p$-value was marginal $(p=0.34)$. On the other hand, disappointingly, when the year of publication increases the laboratory service significantly decreases that needs an incredibly detailed analysis. However, with this limited data, it is impossible to provide tangible evidence to verify this relationship. Regarding publication bias, the funnel plot suggested the presence of minimal evidence of publication bias; however, after conducting the statistical tests, results declare the absence of statistically significant publication bias.

In general, in this review the level of clinical laboratory service satisfaction pooled estimate was unsatisfactory. The year of publication showed a statistically significant relationship with service satisfaction. The primary areas where the customers lacked satisfaction include inadequacy of sitting arrangements, the lack of a secure place to put personal utilities during sample collection, poor latrine hygiene, and extended waiting time to get test results. The health facility administrators particularly service provision supervisors should exert their effort to enhance and maintain their customer satisfaction through strengthening the scope of existing diagnostic services, conducting periodic customer satisfaction surveys, implementing immediate corrective actions for complaints, and having a mechanism of continuous monitoring of laboratory activities. 


\section{Abbreviations}

AJOL: Journals Online, CAPs: College of American Pathologists, ISO: International Organization for Standardization, JCAHO: Joint Commission on Accreditation of Healthcare Organizations, JIB: African Joanna Briggs Institute, QMS: Quality Management System, PRISMA-P: Preferred Reporting Items for Systematic Reviews and Meta-Analysis Protocols

\section{Declarations}

\section{Acknowledgments}

The authors of this review article would like to acknowledge all authors of the primary studies and biomedical staff for providing valuable comments for the successful completion of this review of the review article.

\section{Funding}

This review did not receive any grant.

\section{Availability of data and materials}

All the data generated or analyzed are included in this manuscript and attached as an additional file.

\section{Authors' contributions}

TD and MG conceptualized the draft protocol of the review. All authors searched databases and screened articles based on the eligibility criteria. Three (TD, YM, and TB) authors were involved in the data extraction process. TD and MG contributed to the statistical analysis and interpretation of the results. All authors were equally involved in the write-up of the draft manuscript. TD finalized the manuscript, and all authors read and approved the final manuscript before submission.

\section{Ethics approval and consent to participate}

Not applicable.

\section{Consent for publication}

Not applicable.

\section{Competing interests}

The authors declare that they have no competing interests.

\section{Author details}


${ }^{1}$ University of Gondar, College of Medicine and Health Sciences, School of Biomedical and Laboratory Sciences, Unit of Quality Assurance and Laboratory Management

${ }^{2}$ Department of Medical Microbiology, School of Biomedical and Laboratory Sciences, College of Medicine and Health Sciences, University of Gondar, Gondar, northwest Ethiopia.

\section{References}

1. Assefa F, Mosse A, Hailemichael Y: Assessment of Clients' satisfaction with health service deliveries at jimma university specialized hospital. Ethiop J Health Sci 2011, 21:101-110.

2. Schroeder LF, Amukele T: Medical laboratories in sub-Saharan Africa that meet international quality standards. Am J Cli Pathol 2014, 141:791-795.

3. Gershy-Damet GM, Rotz P, Cross D, Belabbes EH, Cham F, Ndihokubwayo JB, et al: The World Health Organization African region laboratory accreditation process: improving the quality of laboratory systems in the African region. Am J Clin Pathol 2010, 134:393-400.

4. Miller KA, Dale JC: Physicians' satisfaction with clinical laboratory services. Q-Probes (99-03). Northfield, Illinois, College of American Pathologists. 1999:1-10.

5. WHO, WHO Guide for the Stepwise Laboratory Improvement Process Towards Accreditation in the African Region (with checklist),Version 2:2015 For Clinical and Public Health Laboratories.

6. Manzoor F, Wei L, Hussain A, Asif M, Shah SI: Patient Satisfaction with Health Care Services; An Application of Physician's Behavior as a Moderator. Int J Environ Res Public Health 2019, 16:3318.

7. Laohasirichaikul B, Chaipoopirutana S, Combs H: Effective customer relationship of health care: a study of hospitals in Thailand. Journal of Management and Marketing Research 2010, 17:1-12.

8. Nkengasong N: Strengthening laboratory services and systems in resource-poor countries [editorial]. Am J Clin Pathol 2009, 131:774.

9. Mohd A, Chakravarty A: Patient satisfaction with services of the outpatient department. Med J Armed Forces India 2014, 70:237-242.

10. Marquis MS, Davies AR, Ware JE Jr: Patient satisfaction and change in medical care provider: a longitudinal study. Med Care 1983, 21:821-829.

11. Mindaye T, Taye B: Patients satisfaction with laboratory services at antiretroviral therapy clinics in public hospitals, Addis Ababa, Ethiopia. BMC Research Notes 2012, 5:184.

12. Bekele MT: Assessment of patients' satisfaction towards general medical laboratory services at Shenen Gibe Public Hospital, Jimma Town, South West Ethiopia. J Health Med Nurse 2016, 31:7884.

13. Tegbaru B, Meless H, Kassu A, Desalegn T, Gezahegn N, Tamene W, Hailu E, et al: Laboratory services in hospitals and regional laboratories in Ethiopia. Ethiop J Health Dev 2004, 18:43-47.

14. College of American Pathologists: Laboratory accreditation program. Laboratory general checklist.Northfield.GEN.,20335. 2011:1-129. 
15. Guy G, Philip R, David C, El Hadj B, Fatim C, Jean-Bosco N, et al: The World Health Organization African Region Laboratory. Accreditation Process Improving the Quality of Laboratory Systems in the African Region. Am J Clin Pathol 2010, 134:393-400.

16. Abera RG, Abota BA, Legese MH, Negesso AE: Patient satisfaction with clinical laboratory services at Tikur Anbessa specialized hospital, Addis Ababa, ethiopia. Patient Preference and Adherence 2017, $111181-1188$.

17. Hailu HA, Desale A, Yalew A, Asrat H, Kebede S, Dejene D, et al: Patients' satisfaction with clinical Laboratory Services in Public Hospitals in Ethiopia. BMC Health Services Research 2020, 20.

18. Jones BA, Bekeris LG, Nakhleh RE, Walsh MK, Valenstein PN: Physician satisfaction with clinical laboratory services: a College of American Pathologists Q-Probes study of 138 institutions. Arch Pathol Lab Med2009, 133:38-43.

19. Shamseer L, Moher D, Clarke M, Ghersi D, Liberati A, Petticrew M, et al: Preferred reporting items for systematic review and meta-analysis protocols (PRISMA-P) 2015: elaboration and explanation. British Medical Journal 2015, 349:7647.

20. Haylamicheal ID, Desalegne SA: A review of legal framework applicable for the management of healthcare waste and current management practices in Ethiopia. Waste Manag Res 2012, 30:607618.

21. Eshetie S, Gizachew M, Dagnew M, Kumera G, Woldie H, Ambaw F, et al: Multidrug resistant tuberculosis in Ethiopian settings and its association with previous history of anti-tuberculosis treatment: a systematic review and meta-analysis. BMC Infect Dis 2017, 17:219.

22. Federal Democratic Republic of Ethiopia, Health and Health Related Indicators. 2011.

23. Abebe A: Study of hazardous biomedical waste management practices and development of hazardous biomedical waste management guidelines in Addis Ababa. Int J Sci Eng Sci 2017, 1:1932.

24. Feysia B, Herbst CH, Lemma W, Soucat A: The health workforce in Ethiopia: addressing the remaining challenges (English). A World Bank study. Washington, DC: World Bank. 2012.

25. Moher D, Liberati A, Tetzlaff J, Altman DG, Group P: Preferred reporting items for systematic reviews and meta-analyses: the PRISMA statement. PLoS med 2009, 6:e1000097.

26. The Joanna Briggs Institute. Critical Appraisal tools for use in JBI Systematic Reviews Checklist for Prevalence Studies: The University of Adelaide; Available from: http://joannabriggs.org/research/criticalappraisal-tools.html.

27. Nyaga VN, Arbyn M, Aerts M: Metaprop: A stata command to perform meta-analysis of binomial data. Arch Public Health 2014, 72:39.

28. Simonian RD, Laird N: Meta-analysis in clinical trials. Control Clin Trials 1986, 7:177-188.

29. Borenstein M, Hedges LV, Higgins PT, Rothstein HR: Introduction to meta-analysis. John Wiley \& Sons; Borenstein M, Hedges LV, Higgins JP, Rothstein HR. Introduction to meta-analysis. John Wiley \& Sons; 2011 Aug 24. 2009. 
30. George J, Aban IB: An application of meta-analysis based on DerSimonian and Laird method. Journal of Nuclear Cardiology 2016, 23:690-692.

31. Ades AE, Lu G, Higgins JP: The interpretation of random-effects meta-analysis in decision models. Medical Decision Making 2005, 25:646-654.

32. Wang J, Wu X, Lai W, Long E, Zhang X, Li W, et al: Prevalence of depression and depressive symptoms among outpatients: a systematic review and meta-analysis. BMJ Open 2017, 7:e017173.

33. Yeshanew AG, Geremew RA, Temesgen MK: Assessments of patient and health care workers satisfaction on the laboratory services in St. Paul's hospital millennium medical college, Addis Ababa, Ethiopia. International Journal of Scientific Reports 2017 3:192-199.

34. Tesfaye T, Mindaye M, Hassen F, Tesfahun A: Assessments of patients' satisfaction towards clinical laboratory services received at uniformed service hospitals in Addis Ababa, Ethiopia [Thesis (MSc)]. 2014.

35. Teresa M, Bekele S: Assessment of Patients' Satisfaction Towards General Medical Laboratory Services at Shenen Gibe Public Hospital, Jimma Town, South West Ethiopia. Journal of Health, Medicine and Nursing 2016, 31.

36. Teklemariam Z, Mekonnen A, Kedir H, Kabew G: Clients and clinician satisfaction with laboratory services at selected government hospitals in eastern Ethiopia. BMC Research Notes 2013, 6:15.

37. Tefera Z, Tsegaye A, Hassen F: Assessment of Patient satisfaction towards Clinical laboratory services among Strengthening Laboratory Management towards Accreditation (SLMTA) Program Implementing Hospital Laboratories under Addis Ababa City Administration, Ethiopia [Thesis (MSc)]. 2017.

38. Tadele G, Ejeta E, Desalegn M, Abere S, Elias K: Patients Satisfaction on Clinical Laboratory Services at Nekemte Referral Hospital, Oromia, Ethiopia. Food Science and Quality Management 2014, 30:2530 .

39. Mengesha MB: Medical Practitioners' Satisfaction in Southern Ethiopia. Am J Clin Patho/ 2015, 144:895-901.

40. Mekonnen A, Teklemariam Z, Kedir H, Kabew G: Patient Satisfaction with Laboratory Services in Selected Government Hospitals, Eastern Ethiopia. Harar Bulletin of Health Sciences 2011, 1:12-24.

41. Hailu L, Mihiretie H, Dabsu R, Demissie D, Hailu W: Clinician's Satisfaction and Utilization of Laboratory Services in Selected Western Ethiopia Hospitals Nekemte Ethiopia. SCIOL Biomed 2019, 3:136-142.

42. Ejeta E, Tadele G, Desalegn M, Abere S, Elias K: Health care providers' satisfaction with the clinical laboratory service of Nekemte Referral Hospital, Western Ethiopia. International Journal of Medicine and Medical Sciences 2015, 7: 91-97.

43. Desalegn DM, Abay S, Abebe A, Lulie AD, Dejene D, Mersha TB, et al: Quality of Focused Antenatal Care Laboratory Services Provided at Public Health Facilities in Addis Ababa, Ethiopia. Quality in Primary Care 2017, 26 81-89. 
44. Bogale AL, Kassa HB, Ali JH: Patients' perception and satisfaction on quality of laboratory malaria diagnostic service in Amhara Regional State, North West Ethiopia. Malaria Journal 2015, 14:241.

45. Belay M, Abrar S, Bekele D, Daka D, Derbe M, Birhaneselassie M: HIV/AIDS patients' satisfaction on ART laboratory service in selected governmental hospitals, sidamma zone, southern Ethiopia. Science Journal of Public Health 2013, 1:85-90.

46. Alelign A, Belay YA: Patient satisfaction with clinical laboratory services and associated factors among adult patients attending outpatient departments at Debre Markos referral hospital, Northwest Ethiopia. BMC Res Notes 2019, 12.

47. Addis Z, Birhan W, Derseh D, Sahle B, Gizaw N: Physicians' and Nurses' Satisfaction With the Clinical Laboratory Service of Gondar University Hospital, Northwest Ethiopia. Am J Clin Pathol 2013, 140:324-328.

48. Hardy RJ, Thompson SG: Detecting and describing heterogeneity in meta analysis. Stat Med 1998, 17:841-856.

49. Egger M, Smith GD, Schneider M, Minder C: Bias in meta-analysis detected by a simple, graphical test. Bmj 1997, 315:629-634.

\section{Tables}

Table 1: Characteristics of the included studies in the systematic review and meta-analysis for the laboratory customer satisfaction in Ethiopia, 2020. 


\begin{tabular}{|c|c|c|c|c|c|c|}
\hline $\begin{array}{l}\text { First author, } \\
\text { Publication year, } \\
\text { Reference }\end{array}$ & Region & $\begin{array}{l}\text { Study } \\
\text { group }\end{array}$ & $\begin{array}{l}\text { Sampling } \\
\text { technique }\end{array}$ & $\begin{array}{l}\text { Sample } \\
\text { size }\end{array}$ & $\begin{array}{l}\text { Satisfaction } \\
\text { level (\%) }\end{array}$ & $\begin{array}{l}\text { Quality } \\
\text { score }\end{array}$ \\
\hline $\begin{array}{l}\text { Abera RG, } 2017 \\
\text { [16] }\end{array}$ & $\begin{array}{l}\text { Addis } \\
\text { Ababa }\end{array}$ & Patients & Nonprobability & 210 & 60.0 & High \\
\hline $\begin{array}{l}\text { Addis Z, } 2013 \\
\text { [47] }\end{array}$ & Amhara & HPs & $\mathrm{NI}$ & 196 & 51.5 & Low \\
\hline $\begin{array}{l}\text { Aleling A, } 2019 \\
\text { [46] }\end{array}$ & Amhara & Patients & Probability & 391 & 48.3 & High \\
\hline $\begin{array}{l}\text { Belay M, } 2013 \\
\text { [45] }\end{array}$ & SNMP & Patients & $\mathrm{NI}$ & 422 & 90.8 & High \\
\hline $\begin{array}{l}\text { Bogle AL, } 2015 \\
\text { [44] }\end{array}$ & Amhara & Patients & $\mathrm{NI}$ & 300 & 52.7 & High \\
\hline $\begin{array}{l}\text { Desalegn DM, } \\
2017 \text { [43] }\end{array}$ & $\begin{array}{l}\text { Addis } \\
\text { Ababa }\end{array}$ & Pregnant & Probability & 422 & 56.9 & High \\
\hline Ejeta E, 2015 [42] & Oromia & HPs & Probability & 105 & 62.9 & High \\
\hline $\begin{array}{l}\text { Hailu HA, } 2020 \\
\text { [17] }\end{array}$ & Nationwide & Patients & Probability & 2399 & 78.6 & High \\
\hline Hailu L, 2019 [41] & Oromia & HPs & $\mathrm{NI}$ & 207 & 50.7 & Low \\
\hline $\begin{array}{l}\text { Mekonnen A, } \\
2011 \text { [40] }\end{array}$ & $\begin{array}{l}\text { Harari and } \\
\text { Dire Dawa }\end{array}$ & Patients & $\mathrm{NI}$ & 429 & 85.5 & High \\
\hline $\begin{array}{l}\text { Mengesha MB, } \\
2015 \text { [39] }\end{array}$ & SNMP & HPs & Nonprobability & 290 & 75.5 & Low \\
\hline $\begin{array}{l}\text { Mindaye T, } 2012 \\
\text { [11] }\end{array}$ & $\begin{array}{l}\text { Addis } \\
\text { Ababa }\end{array}$ & Patients & Probability & 406 & 85.7 & High \\
\hline $\begin{array}{l}\text { Tadele G, } 2014 \\
\text { [38] }\end{array}$ & Oromia & Patients & Probability & 422 & 60.4 & High \\
\hline $\begin{array}{l}\text { Tefera Z, } 2017 \\
\text { [37] }\end{array}$ & $\begin{array}{l}\text { Addis } \\
\text { Ababa }\end{array}$ & Patients & Nonprobability & 596 & 53.2 & High \\
\hline $\begin{array}{l}\text { Teklemariam Z, } \\
2013[36]\end{array}$ & $\begin{array}{l}\text { Harari and } \\
\text { Dire Dawa }\end{array}$ & Mixed & Probability & 483 & 87.8 & High \\
\hline $\begin{array}{l}\text { Teresa M, } 2016 \\
\text { [35] }\end{array}$ & Oromia & Patients & Nonprobability & 379 & 63.3 & High \\
\hline $\begin{array}{l}\text { Tesfaye T, } 2014 \\
\text { [34] }\end{array}$ & $\begin{array}{l}\text { Addis } \\
\text { Ababa }\end{array}$ & Patients & Probability & 422 & 51.9 & High \\
\hline $\begin{array}{l}\text { Yeshanew AG, } \\
2017 \text { [33] }\end{array}$ & $\begin{array}{l}\text { Addis } \\
\text { Ababa }\end{array}$ & Mixed & Probability & 416 & 57.9 & High \\
\hline
\end{tabular}

Key NI is to represent not indicated 
Table 2: Key findings of satisfaction and dissatisfaction with clinical laboratory services in Ethiopia 


\section{First author, Study Critical indicators of satisfaction and dissatisfaction for the laboratory year of publication group services}

[Reference]

Abera RG, Patients Patients were satisfied with the general hygiene of the laboratory (82\%), 2017 [16] privacy and confidentiality of patient data (83.2\%), and the cost incurred for laboratory services (86.5\%). However, $56 \%, 58.4 \%$, and $63.8 \%$ of the patients were dissatisfied with the location laboratory, availability, and accessibility of latrine, and latrine cleanness and comfort, respectively.

$\begin{array}{lll}\text { Addis Z, } & \text { HPs } & \text { Health workers were dissatisfied with the inconsistency of quality } \\ 2013[47] & \text { laboratory work, absence of timely report for critical values, test } \\ \text { turnaround time, inacceptability of released test results, and the lack of } \\ \text { proper reporting reference range. }\end{array}$

Aleling A, Patients A low proportion $(26.3 \%, 21.3 \%, 21.0 \%, 12.3 \%, 9.7 \%$, and $4.6 \%)$ of 2019 [46] patients have received quality services, preferred service providers, many services, cheap service fee, got the service early, and convenience working hours, respectively. On the other hand, the literacy label of patients, missing laboratory test results, and lack of secure area to put personal utilities during blood collection were indicators statistically associated with the satisfaction level.

Belay $\mathrm{M}, \quad$ Patients $2013[45]$

Patients were satisfied with the language laboratory staff used for communication, hygiene, and attractiveness of the laboratory room, confidentiality of patient results, and the location of the laboratory in the hospital; however, patients were dissatisfied with latrine cleanness, length of waiting time, and lack of adequate information.

Bogle AL, Patients Providing prompt test results, availability of the proper treatment, $2015[44]$ presence of laboratory personnel upon request was among the common enabling factors for patient satisfaction. Whereas, residence, ethnicity, having information about malaria diagnosis after consulting clinician, and test result timeline were the statistically associated predictor variables for satisfaction.
Desalegn
DM, 2017
Pregnant
Considerable (44.8\%) proportion of clients claimed that all ordered
[43] laboratory tests did not require available timely. About $2.6 \%$ of clients missed laboratory investigations due to the unavailability of test request order in the respective antenatal care follow up the center.

\section{Ejeta E, 2015 HPs \\ [42]}

Health professionals were highly satisfied with the location of the laboratory in the hospital, general cleanness of the laboratory rooms, staff courtesy, and improvement of the overall laboratory services. However, they are dissatisfied with the lack of adequate laboratory supplies, the absence of timely reports for critical values, lack of getting urgent test results on time, and inadequacy of test items on the request forms.

Hailu HA, $\quad$ Patients
$2020[17]$

Patients' were dissatisfied with the adequacy of waiting area, blood collection processes (many needlestick attempts), accessibility of sites, long turnaround time, cleanness of latrine, missing of results, communication, availability of requested tests, and cost of the laboratory services.

Hailu L, HPs 2019 [41]

Availability of laboratory reagents, profession, staff respect, result reliability, neatness of the laboratory, availability of request papers in the laboratory, getting urgent test results timely, improvement of laboratory 
services, completeness of laboratory results were predictor variables having a statistically significant association with the outcome variable.

Mekonnen A, Patients Patients were satisfied with the reliability of test results, laboratory $2011[40]$ facilities, and staff performance; however, they were dissatisfied with the latrine sanitation used for sample collection and the lack of explanation on how to follow when using the laboratory services.

\begin{tabular}{|c|c|c|}
\hline $\begin{array}{l}\text { Mengesha } \\
\text { MB, } 2015 \\
{[39]}\end{array}$ & HPs & $\begin{array}{l}\text { Better satisfaction was observed legibility of results, followed by the } \\
\text { manner of the profession, critical value notification, and knowledge of } \\
\text { laboratory professionals. However, less satisfaction was found on the } \\
\text { blood bank services, followed by the availability of senior laboratory } \\
\text { experts in the laboratory. }\end{array}$ \\
\hline
\end{tabular}

Mindaye T, Patients $2012[11]$

Patients who received blood drawing services less than 30 minutes were more satisfied compared to those who underwent for more than 30 minutes. They are dissatisfied with the accessibility and availability of latrines, confidentiality issues, the ability of the phlebotomists, and cleanliness of the blood drawing area.

Tadele G, Patients 2014 [38]

The high level of satisfaction was obtained on the language laboratory workers used for communication and the presence of a waiting place around the laboratory. In contrast, low levels of satisfaction were obtained on the cleanness of latrine and location of the laboratory building in the health facility.

Tefera Z, Patients 2017 [37]

The highest rate of satisfaction was obtained from the location of the laboratory and measures taken to ensure confidentiality during the sample collection process. However, patients were dissatisfied with the adequacy of sitting arrangements at the waiting area, latrine cleanness, and timeline of test results.

Teklemariam Mixed Z, 2013 [36]

Most of the patients were satisfied with the laboratory services. The highest rate of satisfaction was observed on the delivery of prompt test results and the availability of laboratory staff during working hours, respectively. However, the lowest level of satisfaction was identified on critical value notification and cleanness of latrine used for sample collection.

Teresa M, Patients 2016 [35]

Provision of adequate information to collect the specimen, when \& how to receive laboratory test results, respect from laboratory personnel, less than 30 minutes, and between 1 and 2-hours waiting time to receive results were the identified determinant factors to patients' satisfaction with laboratory services.

Tesfaye T, Patients 2014 [34]

Patients were highly satisfied with courtesy by laboratory workers; however, they were highly dissatisfied with the unavailability of laboratory workers during working hours. Significant dissatisfaction determinants were long waiting time to give laboratory specimens, lack of a place to put personal utilities during sample collection in the blood collection area and the latrine, and unavailability of requested test items.

Yeshanew Mixed AG, 2017

The highest level of satisfaction was noted on the courtesy given by laboratory professionals to patients followed by the language of [33] laboratory professionals used to communicate patients and the cost of laboratory services. However, the least level of patient satisfaction rate was found on the location and size of the laboratory, waiting for the place, and the cleanness of the latrine used for sample collection. 
Table 3: Subgroup analysis of predictor variables to the satisfaction of clinical laboratory services in Ethiopia.

\begin{tabular}{|c|c|c|c|c|c|}
\hline Variables & Variable category & $\begin{array}{l}\text { Satisfaction level } \\
(\%)\end{array}$ & $\begin{array}{l}95 \% \\
\mathrm{Cl}\end{array}$ & p.value & $\mathrm{I}^{2}(\%)$ \\
\hline \multirow[t]{2}{*}{ Sample size } & Below 384 & 60 & $53-67$ & $\leq 0.001$ & 88.82 \\
\hline & 384 and above & 70 & $60-79$ & $\leq 0.001$ & 98.56 \\
\hline \multirow[t]{3}{*}{ Publication year } & 2011-2013 & 82 & $71-90$ & $\leq 0.001$ & 96.80 \\
\hline & $2014-2016$ & 61 & $54-68$ & $\leq 0.001$ & 90.36 \\
\hline & $2017-2020$ & 58 & $46-70$ & $\leq 0.001$ & 98.18 \\
\hline \multirow{3}{*}{$\begin{array}{l}\text { Sampling } \\
\text { technique }\end{array}$} & Probability & 67 & $56-76$ & $\leq 0.001$ & 98.24 \\
\hline & Non probability & 63 & $53-73$ & $\leq 0.001$ & 93.19 \\
\hline & NA & 68 & $48-85$ & $\leq 0.001$ & 98.54 \\
\hline \multirow[t]{3}{*}{ Study participants } & Patients & 67 & $57-75$ & $\leq 0.001$ & 98.30 \\
\hline & $\begin{array}{l}\text { Health } \\
\text { professionals }\end{array}$ & 60 & $47-73$ & $\leq 0.001$ & 93.22 \\
\hline & Mixed & 75 & $73-78$ & - & - \\
\hline \multirow[t]{2}{*}{ Study quality } & High quality & 66 & $57-74$ & $\leq 0.001$ & 98.08 \\
\hline & Low quality & 52 & $47-85$ & $\leq 0.001$ & 98.04 \\
\hline
\end{tabular}

Key *NA stands for not indicated

Figures 


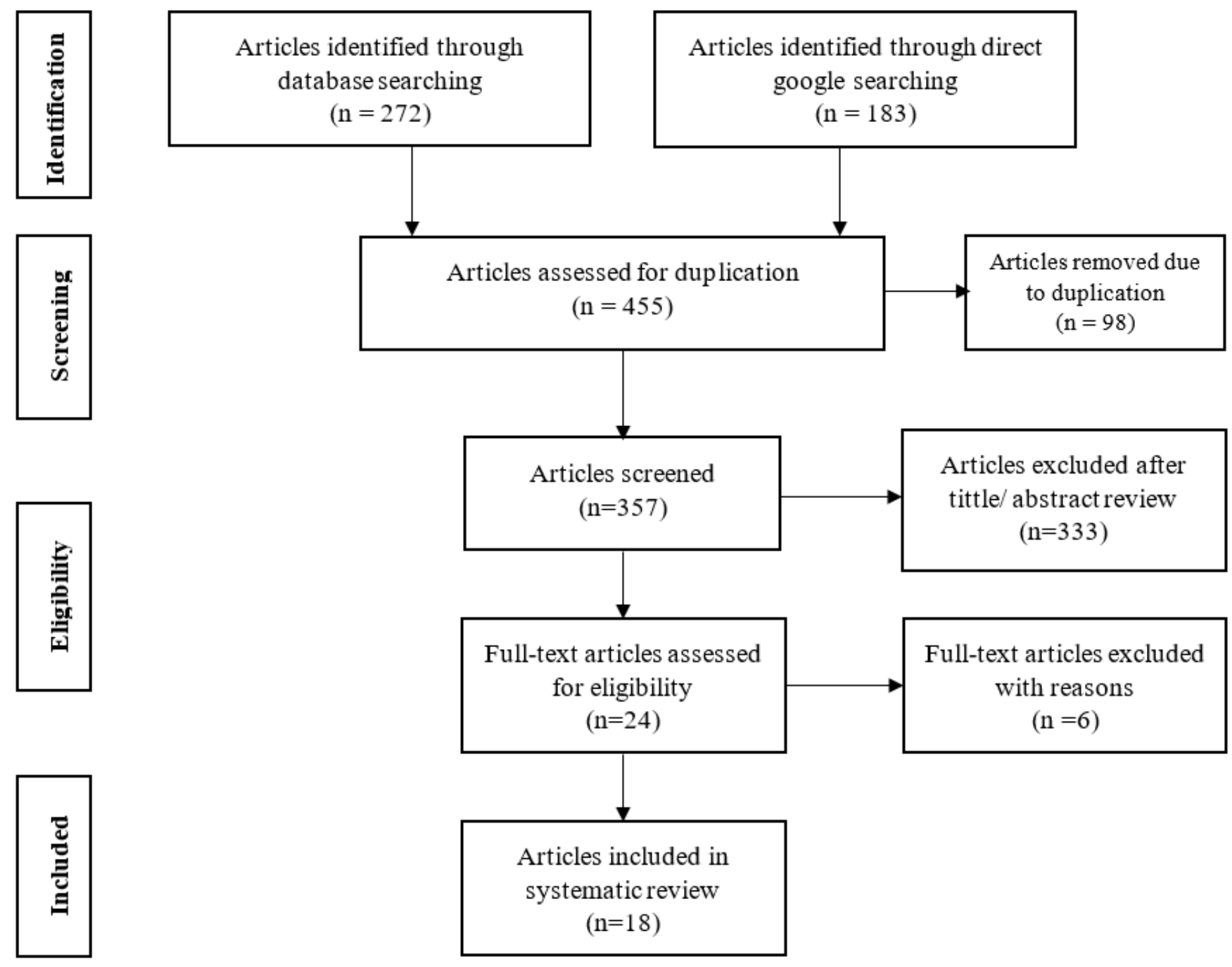

Figure 1

PRISMA flow diagram for identification and selection of articles for inclusion in the review. 
Study

Abera RG. (2017)

Addis Z. (2013)

Alelign A. (2019)

Belay M. (2013)

Bogale AL. (2015)

Desalegn DM. (2017)

Ejeta E. (2015)

Hailu HA. (2020)

Hailu L. (2019)

Mekonnen A. (2011)

Mengesha MB. (2015)

Mindaye T. (2012)

Tadele G. (2014)

Tefera Z. (2017)

Teklemariam Z. (2013)

Teresa M. (2016)

Tesfaye T. (2014)

Yeshanew AG. (2017)

Overall $\left(\mathrm{I}^{\wedge} 2=97.97 \%, \mathrm{p} \leq 0.01\right)$
$\mathrm{ES}(95 \% \mathrm{CI})$

\% Weight

5.48

5.46

$0.48(0.43,0.53) \quad 5.58$

$\checkmark \quad 0.91(0.88,0.93) \quad 5.59$

$0.53(0.47,0.58) \quad 5.55$

$0.57(0.52,0.62) \quad 5.59$

$0.63(0.53,0.72) \quad 5.26$

$0.79(0.77,0.80) \quad 5.69$

$0.51(0.44,0.58) \quad 5.47$

$0.86(0.82,0.89) \quad 5.60$

$0.76(0.70,0.80) \quad 5.54$

$0.86(0.82,0.89) \quad 5.59$

$0.60(0.56,0.65) \quad 5.59$

$0.53(0.49,0.57) \quad 5.63$

- $\quad 0.88(0.85,0.91) \quad 5.61$

$0.63(0.58,0.68) \quad 5.58$

$0.52(0.47,0.57) \quad 5.59$

$0.58(0.53,0.63) \quad 5.59$

$0.66(0.59,0.73) \quad 100.00$

\section{$\begin{array}{ccccc}\mid & \mid & \mid & \mid & \text { | } \\ 0 & .25 & .5 & .75 & 1\end{array}$}

Proportion (p)

Figure 2

The proportion of customer satisfaction rate with clinical laboratory services in Ethiopia. 


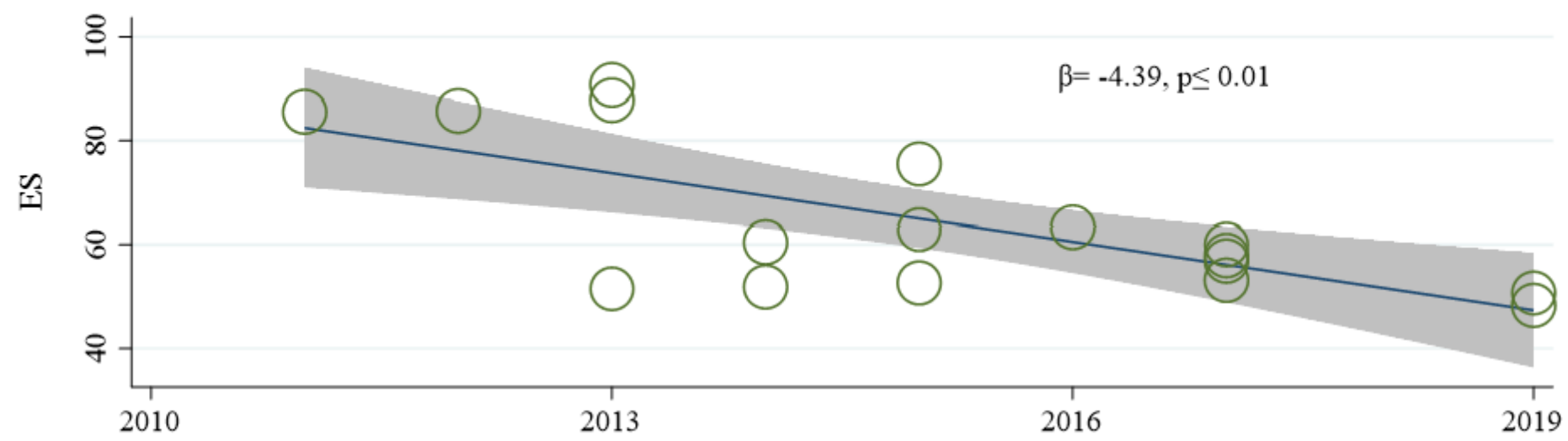

Fig. A

Year of publication

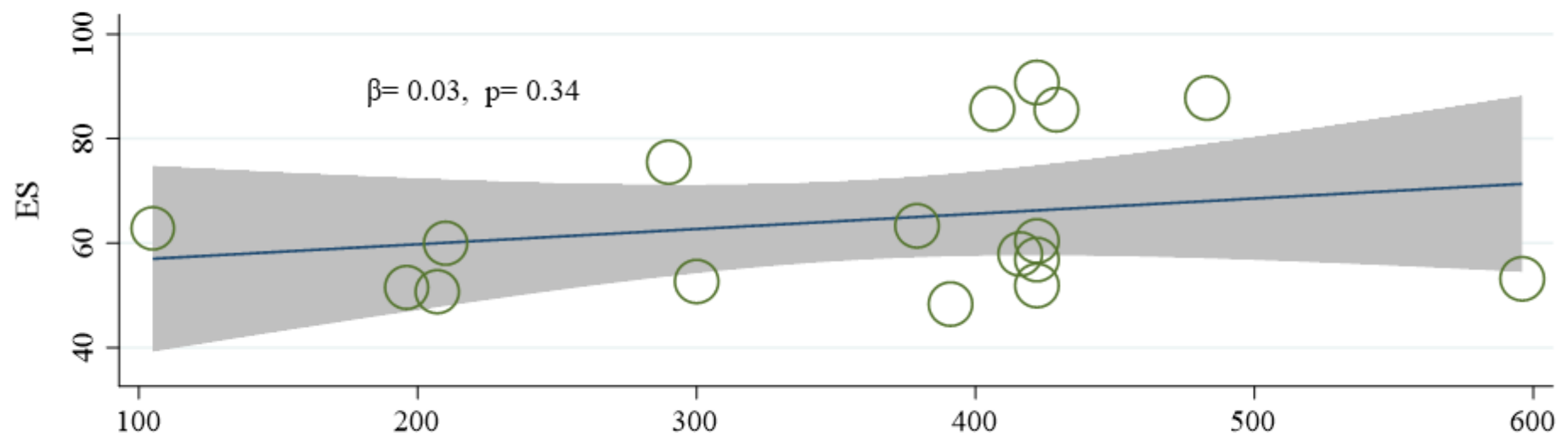

Fig. B

Sample size

\section{Figure 3}

A meta-regression analysis of customer satisfaction with clinical laboratory services based on selected studies' publication years. The slopes of the regression lines indicate either increasing or decreasing the effect estimate using REML estimation. Grey colour around the slope of the regression line indicates the 95\% confidence interval. Each circle represented a single study, and its size corresponds to the weight assigned to each study. 
Meta-analysis estimates, given named study is omitted

| Lower CI Limit

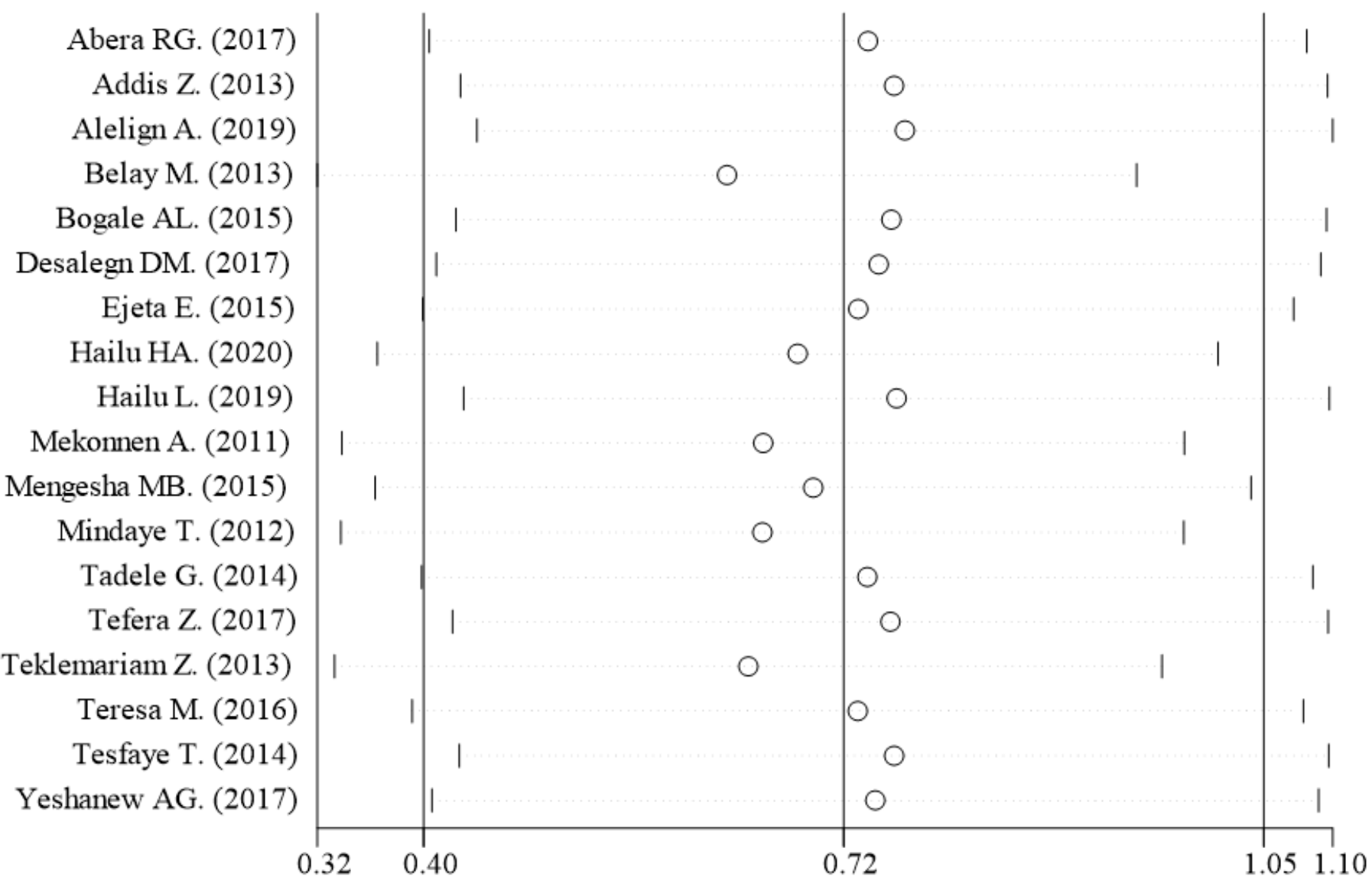

\section{Figure 4}

Sensitivity analysis of level laboratory customer satisfaction with clinical laboratories in Ethiopia. 


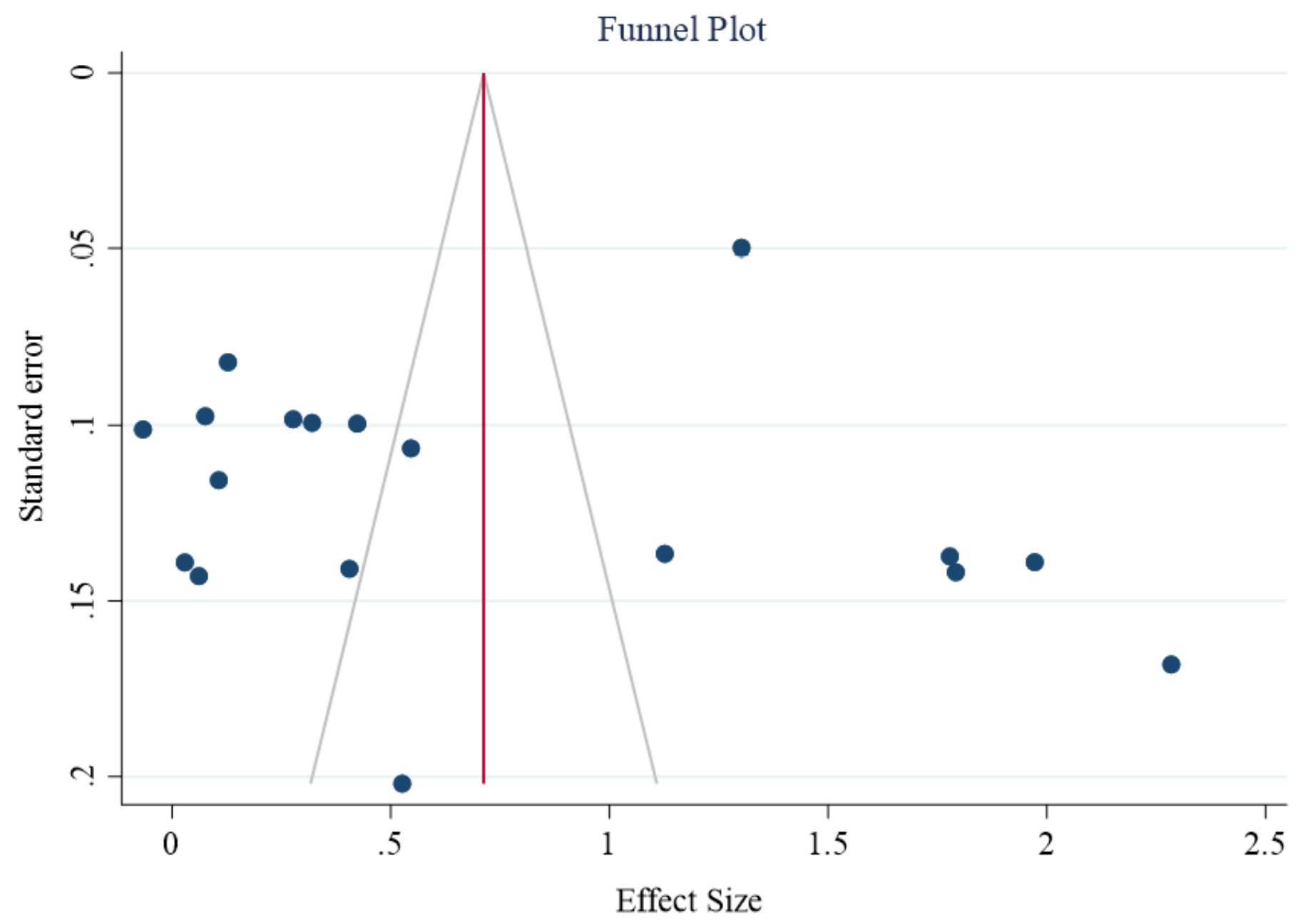

Figure 5

Funnel plot of studies on the level of customer satisfaction with clinical laboratory services in Ethiopia.

\section{Supplementary Files}

This is a list of supplementary files associated with this preprint. Click to download.

- Additionalfile1.MedlineSearchStrategy.docx 